\title{
Sistema de Inteligência Acadêmica na Gestão de Pós-Graduação Stricto Sensu em Administração
}

\author{
Daniela Luiza de Macedo ${ }^{1}$ \\ Leonel Cezar Rodrigues ${ }^{2}$ \\ Amélia Silveira ${ }^{3}$
}

\section{Resumo}

O objetivo deste trabalho foi analisar a gestão de dois Programas de Pós-Graduação Stricto Sensu em Administração (PPGSSA) buscando contribuir com proposições para a implantação de um Sistema de Inteligência Acadêmica (SIAc), como apoio aos processos decisórios e à formulação de estratégias nos Programas pesquisados. Os PPGSSAs selecionados estão vinculados a Instituições de Ensino Superior (IES), uma pública e outra privada, tendo sido selecionados de forma intencional em razão de critérios de qualidade. O referencial teórico envolveu, principalmente, a literatura sobre Inteligência Competitiva (IC) e suas interfaces com a formulação de estratégias. A pesquisa foi empírica, exploratória, com método qualitativo, e de estudo de casos múltiplos. Os resultados indicam que em ambos os Programas há indícios de utilização de Inteligência Acadêmica (IA), ainda que de forma simplificada e orientada para responder aos requisitos de qualidade indicados pela CAPES. A análise permitiu apontar o papel e as funções para um SIAc, dentro da estrutura administrativa e operacional dos programas estudados, na qual este suporta e aperfeiçoa a formulação de estratégias para a melhoria da qualidade, com aumento da visibilidade e da inserção social dos dois programas, objeto de análise. Há compreensão e entendimento da oportunidade da adoção de um SIAc nos PPGSSAs pelos gestores, líderes de linha de pesquisa e pelos especialistas técnicos participantes da pesquisa.

1 Mestre em Administração pela Universidade Nove de Julho-UNINOVE. End.: Av. Francisco Matarazzo, 612, São Paulo - SP. CEP: 05001-100 - Brasil. E-mail: danyluma@gmail.com.

2 Doutor em Administração pela Vanderbilt University, TN (USA). Professor do Programa de Mestrado e Doutorado em Administração - PMDA da Universidade Nove de Julho - UNINOVE. End.: Av. Francisco Matarazzo, 612, São Paulo - SP. CEP: 05001-100 - Brasil. E-mail: leonelcz@gmail.com.

3 Doutora em Ciências da Comunicação pela Universidade de São Paulo - USP. Professora do Programa de Mestrado Profissional em Gestão e Práticas Educacionais da Universidade Nove de Julho - UNINOVE. End.: Av. Francisco Matarazzo, 612, São Paulo - SP. CEP: 05001-100 - Brasil. E-mail: amelia@floripa.com.br.

Artigo recebido em: 04/08/2010. Aceito em: 07/05/2011. Membro do Corpo Editorial Científico responsável pelo processo editorial: Thomas G. Brashear.

(c) (i) (9) Esta obra está sob uma Licença Creative Commons Atribuição-Uso. 
Palavras-chave: Inteligência Competitiva. Gestão Estratégica da Pós-Graduação. Inteligência Acadêmica. Sistema de Inteligência Acadêmica (SIAc).

\section{Introdução}

Antecedentes históricos relatam a influência de estrutura militar na concepção da administração. Assim, a rígida estrutura linear, a unidade de comando e escala hierárquica presentes nas unidades militares mantiveram-se nos estudos de perspectiva clássica da administração, tendo Frederik W. Taylor, Henry Ford, Henri Fayol e Max Weber como seus principais condutores. A contribuição desses estudos foi primordial para o 'nascimento' da administração como obra distinta, disciplina escolar e área de estudo no início do Século XX (DRUCKER, 1984).

Cabe notar que assim como a administração, também, a Inteligência Competitiva (IC) tem em seus antecedentes históricos traços da influência militar. Historicamente, com o fim da Guerra Fria, as pessoas que trabalhavam para os serviços de espionagem de diversas organizações governamentais encontraram nas empresas um campo que se beneficiaria de suas aptidões específicas de práticas e conhecimentos da inteligência, para coletar e tratar as informações de forma ética e legal (GOMES; BRAGA, 2006). Essa maneira de coletar e tratar as informações são revelados na literatura sobre IC, que apresenta sua contribuição para estruturar o gerenciamento e a coordenação das atividades organizacionais, principalmente por meio de um Sistema de Inteligência Competitiva (SIC). Um SIC pode contribuir para a organização em dois aspectos: a tomada de decisão e a formulação da estratégia. Segundo Silva (2005), essa contribuição se dá por meio de um processo coordenado de coleta, análise e disseminação de informações, tendo como usuários finais os tomadores de decisão e os formuladores de estratégia das mais diversas organizações.

No contexto específico de uma organização acadêmica, mais precisamente da Pós-Graduação Stricto Sensu, todas as decisões e a estratégia de seus gestores estão diretamente relacionadas com o alcance de um objetivo: a classificação de seus cursos em nível de excelência pela Coordenação de Aperfeiçoamento de Pessoal de Nível Superior (CAPES), em uma escala de sete níveis, no qual o mais elevado corresponde ao maior grau de excelência. A CAPES, ao longo do 
tempo, tem sido o órgão responsável pela determinação dos padrões e da forma de avaliação da qualidade dos Programas de Pós-Graduação Stricto Sensu, no Brasil, visando garantir sua expansão e consolidação. Como as avaliações da CAPES são realizadas periodicamente, cabe aos gestores de Programas de Pós-Graduação Stricto Sensu utilizar-se de instrumentos para atingir este nível de excelência, como um objetivo. Para tanto, se fazem necessárias a formulação e a implantação de estratégias para gerir com eficiência os Programas, com o intuito de alcançar o objetivo de classificação do curso no mais alto grau de excelência na CAPES.

Argumenta-se que, nesse contexto, há possibilidade de adoção do SIC como mecanismo para vencer barreiras e assegurar a excelência na gestão dos Programas de Pós-Graduação Stricto Sensu. Nesse caso, o SIC passará a ser denominado de Sistema de Inteligência Acadêmia (SIAc), tomando como base as diretrizes do SIC, porém este voltado para uma abordagem acadêmica. Um SIAc contribuirá para a formulação e implementação das estratégias delineadas pelos PPSS.

Com esse entendimento, o objeto de estudo voltou-se para o ambiente de dois Programas de Pós-Graduação Stricto Sensu em Administração, sendo um pertencente a uma IES pública, e o outro a uma IES privada, onde ambas estão localizadas na capital de São Paulo. Assim, o objetivo central do estudo buscou analisar a gestão de dois PPGSSAs, em São Paulo, SP, buscando contribuir com proposições para a implantação de um SIAc, como apoio aos processos decisórios e à formulação de estratégias nos Programas pesquisados. Para tanto, os objetivos específicos identificaram o formato de gestão desses dois Programas de Pós-Graduação em Administração selecionados, segundo a percepção dos sujeitos sociais selecionados, e investigaram a presença $e$ as possíveis contribuições da inteligência acadêmica na gestão dos Programas selecionados.

O artigo foi estruturado em cinco seções, incluindo essa introdução. A segunda parte apresenta uma revisão sobre Inteligência Competitiva e seus ciclos, processos e sistemas, assim como a relação com o Planejamento Estratégico e a Estratégia. A terceira descreve os procedimentos metodológicos adotados, sendo que a quarta seção apresenta os resultados da pesquisa. A quinta e última seção tece considerações finais e elenca as principais conclusões do estudo. 


\section{Inteligência Competitiva: conceituação}

Devido à herança militar que marcou a IC no campo da administração, a opinião pública continua, ainda que em parte, a relacionar o termo "inteligência" com clandestinidade, informações confidenciais, agentes secretos e espionagem (PRESCOTT; MILLER, 2002). Dessa forma, nos primeiros anos de transição da IC para o mundo empresarial, artigos e reportagens sobre informação e vantagens competitivas abordaram jocosamente a IC pelo lado do vazamento de informação, subornos, coerção e espionagem industrial entre outros (MILLER, 2002). É importante elucidar que apesar de uma maior compreensão acerca do papel da IC nas decisões e na formulação de estratégias, a IC na empresa não se fundamenta em atos de espionagem, roubo ou furto de informações. O objeto de informações da IC são fontes públicas e livres de dados e informações que podem ser usados, de forma independente de sua extensão, em beneficio da estratégia competitiva das empresas (FULD, 1995). Combs (1992) define a IC como um processo analítico que retrata a posição, o desempenho, as potencialidades e as intenções dos concorrentes $e$ que transforma a informação em inteligência relevante para o conhecimento estratégico. Combs (1992) ressalta elementos como informação inteligente e conhecimento estratégico no conceito da IC, o que enriquece sua função e o papel desempenhado nos processos de negócio.

Sob o ponto de vista do conceito de IC, a Society of Competitive Intelligence Professionals (SCIP) define IC como um programa sistemático, contínuo legal e ético para reunir, analisar e administrar informações externas que podem afetar planos, decisões e operações de uma empresa (SCIP, 2010). Com esse mesmo entendimento, Comai (2004) e Coelho et al. (2006) veem a IC como um processo sistemático de recolher, classificar, analisar, gerir e disseminar a informação sobre os ambientes competitivos do mercado concorrencial e organizacional. Convém esclarecer que se utilizando de práticas éticas e legais, este deve ser integrado no processo de tomada de decisão para atingir as metas estratégicas da organização. Por sua vez, a Associação Brasileira dos Analistas de Inteligência Competitiva (ABRAIC) entende a IC como um processo informacional proativo que direciona os responsáveis na organização para melhor tomada de decisão, seja ela estratégica ou operacional (ABRAIC, 2010).

O objeto da IC é a informação relevante para o negócio e, nesse processo, o objetivo não é roubar os segredos de propriedade dos concorrentes, mas sim 
recolher informação de maneira legal e sistemática, montando uma base de informações em larga escala que, ao serem ordenados e analisados, fornecem uma compreensão mais ampla da estrutura, cultura, comportamento, potencialidades e fraquezas da empresa concorrente (COMBS, 1992; WEISS, 2002; TYSON, 2002). O produto final da IC é a informação processada e orientada para os interesses dos tomadores de decisão que permita à empresa estar à frente de seus concorrentes (COELHO et al., 2006).

Dada a crescente importância da IC nas organizações, Coelho et al. (2006) identificaram um expressivo crescimento das práticas de IC na última década, devido não apenas à sua relevância nas empresas, mas também à multiplicação das fontes públicas de informação científicas, técnicas e econômicas. Para Miller (2002) e Fuld (1995), a IC não é exclusiva das grandes empresas multinacionais. Pelo contrário, a IC é de igual utilidade para as organizações de pequeno e médio porte, visto que também precisam construir vantagens competitivas.

O papel e funções da IC nas organizações, e especialmente visões diferentes de suas finalidades, têm levado os estudiosos deste campo do conhecimento a avaliar a IC sob os diferentes ângulos. Fleisher et al. (2007) realizaram um extenso trabalho de garimpagem dos principais termos e significados utilizados em contextos distintos, mas com mesmos objetivos, como por exemplo: competição, análise e ética de negócios; benchmarking; perfil competitivo; pesquisa $e$ análise de mercados.

A natureza constitutiva da IC envolve elementos essenciais em sua constituição, tais como seus processos e ciclos, no processo de inteligência competitiva.

\subsection{Processo de Inteligência Competitiva}

Como os tomadores de decisões precisam estar bem informados sobre questões fundamentais que envolvem o negócio da empresa, é necessário um processo ou ciclo de inteligência formal e sistemático. Herring (1999) propôs um ciclo do processo de IC de quatro estágios, que mais tarde é retomado e ampliado por Miller (2002), transformando-o em cinco estágios. Para melhor entendimento do que foi proposto por Herring (1999), e retomado e ampliado por Miller (2002), apresenta-se, a seguir, os cinco estágios do processo ou ciclo de inteligência competitiva: Planejamento e direção - define-se a concentração da coleta de dados e sua execução. Identifica as principais pessoas interessadas no assunto que podem ser os responsáveis pelas decisões em toda a empresa; 
Coleta - é composto pelo recolhimento e categorização dos dados sobre fatos relativos ao ambiente externo de uma empresa, constituindo-se em informações; Análise - analisar e sintetizar as informações para a criação da inteligência; Disseminação - disseminar a informação entre os responsáveis pelas decisões; Monitoramento - controlar ou manter atenção permanente às fontes cognitivas externas e internas, para prevenir tendências ou corrigir desvios.

Para Correia (2006), o processo ou ciclo da IC é planejado e construído a partir da identificação das necessidades de informação pela organização ou pelos setores específicos. Tomando como base Rodrigues e Riccardi (2007) e Rodrigues (2010), a Figura1mostra que a identificação das necessidades de informação pode ser influenciada pelo modelo de negócio da organização, bem como pelas políticas e diretrizes corporativas, processos administrativos e das tecnologias e inovação que envolve o ambiente organizacional. Dessa forma, após a identificação das necessidades é preciso planejar o trabalho a ser realizado pela IC. Ou seja, para responder aos demandantes da necessidade deve-se buscar a informação que eles necessitam. A sondagem (prospecção) para a coleta dos dados necessários deve ser feita nas bases cognitivas públicas e humanas. Coletados os dados, a seguinte etapa será a de análise dos mesmos para seleção dos cabíveis ou úteis como respostas às necessidades explicitadas inicialmente. O processo de análise e seleção leva à geração de um produto da equipe de IC. Então, o produto deve ser disseminado pela área de IC aos demandantes das necessidades. Quando utilizados pelos demandantes, tais dados se transformam em informações, que normalmente, aumentam o nível de conhecimento e a expertise deles. Sempre que os demandantes identificarem novas necessidades de informação, o processo de IC executará o ciclo das etapas novamente, gerando o conhecimento desejado pelos demandantes. 


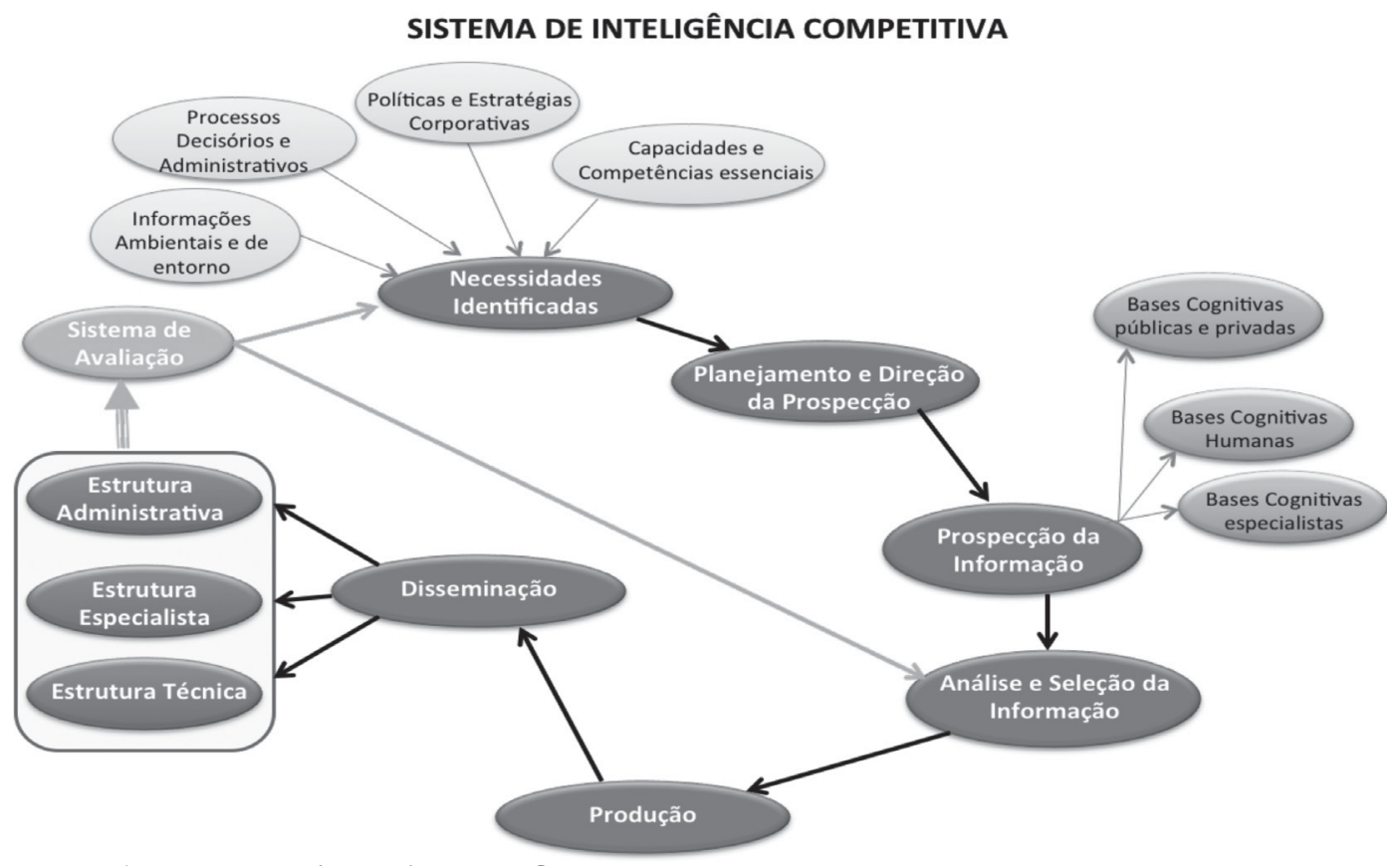

Figura 1: Processos de Inteligência Competitiva Fonte: Rodrigues (2010, p. 180)

A IC fará parte integrante do Sistema de Inteligência Competitiva (SIC), que por sua vez permitirá que as informações cheguem de forma rápida e segura para todos os níveis da organização, reduzindo, assim, incertezas e riscos de insucesso nas decisões tomadas (BALESTRIN, 2001).

\subsection{Sistema de Inteligência Competitiva (SIC)}

Um sistema de inteligência deve identificar primeiramente, os tipos vitais de informações competitivas e as suas melhores fontes. Depois o sistema deverá, continuamente, coletar informações do campo e de dados publicados. Em seguida, deve avaliar a validade e a confiabilidade da informação, interpretá-la, organizá-la de forma apropriada e, para concluir, enviar as melhores informações para os diversos níveis organização com a finalidade de apoiar tomadas de decisão mais acertadas (KOTLER; ARMSTRONG,1993). De acordo com Fuld (1995), o SIC não é apenas um conjunto de técnicas de coleta que visa responder a uma única questão específica, mas sim, um conjunto de processos sistematizados que organiza todos os tipos de informações coletadas. Bessa 
(2007) explica que um SICs caracteriza-se por ser um mecanismo informacional, um processo sistemático pró-ativo, que envolve: a) coleta e busca ética de dados, formais e informais, abrangendo as entidades e forças do macro ambiente, do ambiente competitivo e do ambiente interno da empresa; b) análise depurada e integrada das informações e; c) disseminação das informações.

Quanto à elaboração de um SIC na organização é necessária uma estrutura de apoio ao seu funcionamento, gerenciamento e coordenação (SAMMON; KURLAND; SPITALNIC, 1984) como a disponibilização de recursos financeiros, humanos e de informática (BATTAGLIA, 1999). Segundo Kahaner (1996), a organização deve elaborar uma estrutura que seja responsável pela estrutura e funcionamento da SIC. Kahaner (1996) denomina essa estrutura como "funcional-burocrática". O objetivo da estrutura funcional-burocrática é o suprimento adequado de informação aos tomadores de decisão. A estrutura funcional-burocrática deve contar: a) com um gestor que deverá ter o apoio da alta direção da organização e manter uma relação direta com seus membros e; b) com usuários-chave que provavelmente serão os tomadores de decisão da organização, localizados ao longo dos níveis operacionais, táticos e estratégicos.

\subsection{Inteligência Competitiva e a Interação com o Planejamento Estratégico e Estratégia}

Buscando ainda um melhor entendimento no assunto convém ressaltar que, durante algumas décadas, as organizações elaboravam planejamentos para conduzirem os seus negócios por dez ou vinte anos. Porém, nos dias atuais, face à redução dos ciclos de vida de produtos, tecnologias e mesmo de mercados, o tempo de um plano estratégico mantém-se entre três a cinco anos. Por mais que o planejamento estratégico nas organizações busque o desenvolvimento de soluções a médio e longo prazo (ZACCARELLI, 2002), faz-se necessário rever suas diretrizes periodicamente, numa tentativa de mantê-lo atualizado a fim de aproveitar as oportunidades e reduzir riscos de insucesso do negócio. Segundo a definição de Fischmann e Almeida (1991, p. 25), entende-se que planejamento estratégico como:

[...] uma técnica administrativa que, através da análise do ambiente de uma organização, cria a consciência das suas oportunidades e ameaças, dos seus pontos fortes e fracos para 
o cumprimento da sua missão e, através desta consciência, estabelece o propósito de direção que a organização deverá seguir para aproveitar as oportunidades e reduzir riscos.

Convém também ressaltar que o bom desenvolvimento do planejamento estratégico pede que princípios norteadores das organizações com omissão, visão, objetivos, crenças e valores estejam bem definidos. Uma vez definidos estes princípios é possível identificar os principais pontos fortes e fracos e depois fazer uma avaliação dos mesmos, verificando o que pode ser superado ou melhorado. Dessa maneira, é possível elaborar um planejamento consistente que então, determina o que deve ser feito para se cumprir os objetivos estratégicos estabelecidos. Segundo Almeida (2003) diversos são os modelos de planejamento estratégico, sendo que cada um possui peculiaridades e características específicas. Mas, praticamente, todos seguem a mesma lógica: diagnóstico do ambiente, seleção da estratégia e o planejamento de o que deve ser feito para se cumprir os objetivos estratégicos estabelecidos.

Por sua vez, entende-se a estratégia como instrumento de gestão administrativa que aloca eficientemente recursos, alinhando com os processos constituídos para atingir um determinado objetivo nas organizações. Mintzberg (2001), um dos grandes estudiosos sobre os aspectos que envolvem a estratégia, afirma que a estratégia pode ser definida como uma força mediadora entre a organização e o seu meio envolvente: um padrão de orientação organizacional no processo de tomada de decisões organizacionais. Mintzberg e Quinn (2001) também apresentam o conceito de estratégia como posicionamento mercadológico dos negócios das organizações.

Convém enfatizar que uma das definições apresentadas anteriormente por Porter (1996) afirma que a estratégia é o encontro de uma posição vantajosa em relação aos concorrentes de uma organização, que compreende a anulação de forças do entorno competitivo além e ajustes internos em seus processos. Porter (1996) alerta, entretanto, que a estratégia não é equivalente à eficácia operacional de uma organização. Para esse autor, a estratégia deve distinguir uma empresa de seus concorrentes, seja em diferenciação, seja em foco (segmento), ou seja, em custo. A organização deve elaborar um conjunto diferente de atividades para proporcionar um mix único de valores, criando assim, uma posição exclusiva e valiosa no mercado. (PORTER,1999). Para Connolly et al. (2005, p. 153), a estratégia pode ser vista como o "[...] planejamento central 
utilizado por uma organização para identificar e atingir os seus objetivos e gerir os aspectos mais fundamentais de uma empresa: quem ela é e o que faz".

\subsection{Adoção do SIC para Elaboração do Planejamento Estratégico e da For- mulação de Estratégias}

Juntamente com o planejamento estratégico que apresenta o que deve ser feito para alcançar resultados consolidados, a estratégia também pode ser desenvolvida por profissionais de setores específicos da organização, mas sempre se direcionando em benefício dos objetivos organizacionais. No entanto, os profissionais que desenvolvem o planejamento e a estratégia devem ter total confiança nas informações, que são base para a elaboração do planejamento e a formulação de estratégias de qualidade e assim, direcionar os gestores à tomada de decisão mais acertada e oportuna. Por isso, a organização que possuir a área de IC poderá utilizar-se da competência dessa área para sustentar o planejamento estratégico e a formulação de estratégias. Informações confiáveis facilitarão o processo de tomadas de decisão, ajudando a construção de estratégias mais eficazes para a organização (MILANO, 2004).

Complementando Milano (2004), Biagi e Berjeaut (2006, p. 49) destacam que o trabalho desempenhado pelo SIC nas organizações tem o claro propósito de fornecer aos executivos das organizações, o conhecimento sobre "[...] as atividades dos competidores, as preferências dos consumidores e as tendências de inovação no tempo em que aparecem". Ainda de acordo com esses autores, com as atividades do SIC, os executivos podem evitar a tomada de decisão baseada apenas em intuição ou suposições aumentando o risco de erro e diminuindo a sua eficácia.

A Figura 2 apresenta a importância da função do SIC para o planejamento estratégico e a formulação de estratégias. A participação do SIC com os principais elementos de atuação de gestão (planejamento estratégico e formulação de estratégias) é uma maneira de fornecer informações sólidas sobre o entorno competitivo da organização ou do setor. O SIC pode ser suporte para a fase de diagnose do planejamento estratégico. Nessa fase, o SIC pode contribuir para a identificação dos aspectos internos relevantes nas organizações, na identificação do real campo de atuação e na busca das informações ambientais nas bases cognitivas públicas (do macro ambiente clima, solo e operacional), os quais podem ter influência sobre o desempenho da organização. Com o planejamento 
estratégico devidamente elaborado e após definição dos objetivos estratégicos, a próxima etapa é a formulação de estratégias. Essa etapa também pode contar efetivamente com o SIC. A identificação das necessidades pelos elaboradores de estratégias (que podem ser os estrategistas organizacionais ou gestores de setores específicos) será demandada à área de IC que, realizará o seu ciclo de atividades, a fim de disseminar aos demandantes um produto que contenha informações consistentes, capacitando os demandantes da necessidade com o conhecimento apropriado para elaborar estratégias eficazes.

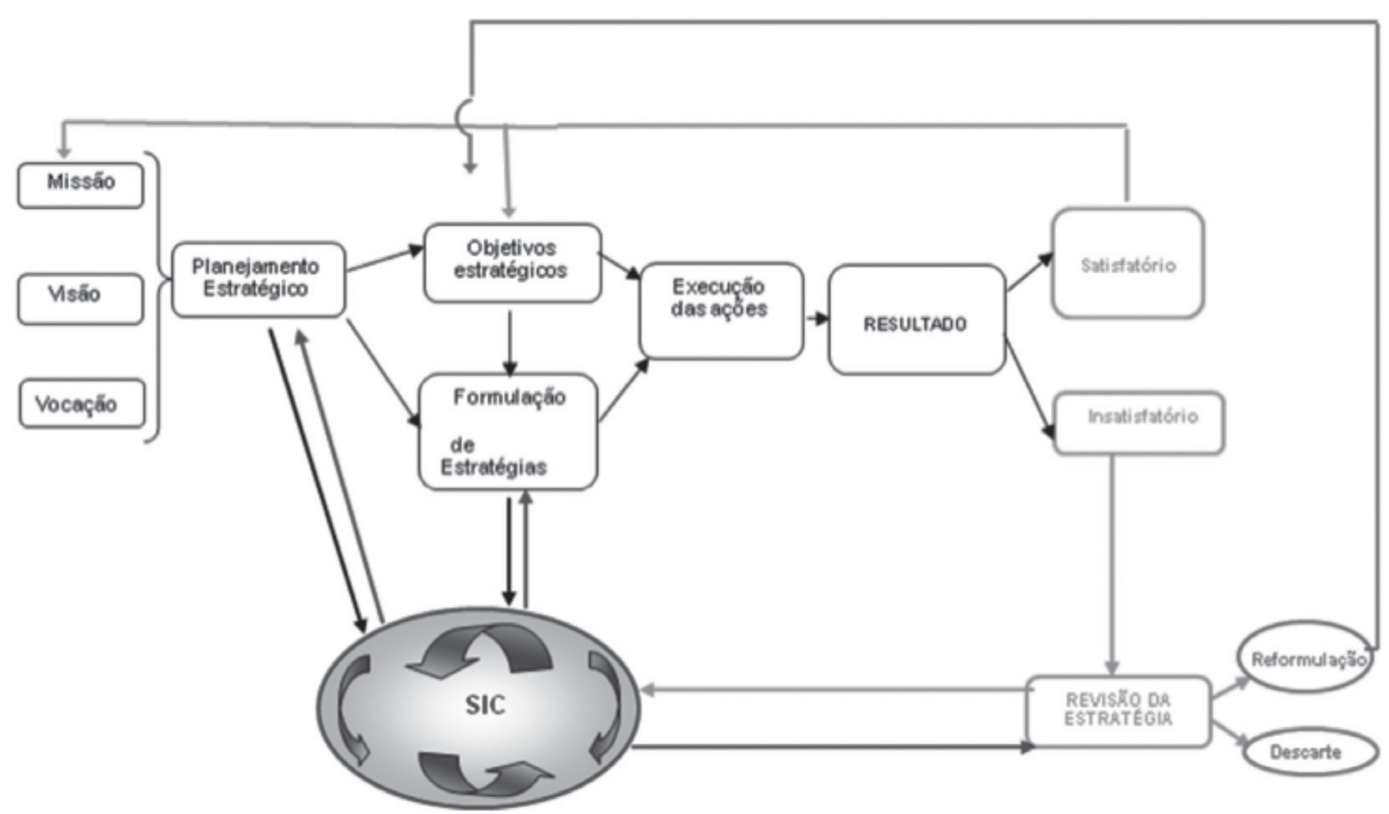

Figura 2: A função do SIC para o planejamento estratégico e a formulação de estratégias Fonte: Elaborada pelos autores deste artigo com base nos dados de pesquisa

Essas estratégias direcionarão a tomada de decisão (execução das ações). Assim, a tomada de decisão corresponderá a um resultado, que poderá ser satisfatório ou insatisfatório. Nota-se que o resultado satisfatório atinge os objetivos estratégicos (organizacional ou setorial), estando de acordo com a missão, visão e vocação organizacional.

No entanto, um resultado não satisfatório pede que a estratégia seja revista. Então, novamente, a necessidade identificada volta ao SIC, que desenvolverá seu trabalho, a fim de encontrar as informações pertinentes. O SIC iniciará um novo ciclo que então, deverá fornecer aos demandantes as informações 
corretas para a melhor estruturação das suas atividades. Com o conhecimento necessário, os demandantes das informações poderão reformular a estratégia ou, se for o caso, descartá-la completamente. Portanto, para evitar erros no planejamento estratégico, na formulação de estratégias e na tomada de decisão uma boa área de IC que esteja ligada diretamente aos gestores pode ser extremamente útil.

Com relação a um setor específico, os objetivos setoriais devem estar alinhados e contribuir para a consecução dos objetivos organizacionais, em que o SIC pode ser utilizado como mecanismo capaz de suprir as organizações de informações relevantes que garantirão o sucesso da implantação de estratégias eficazes.

No âmbito da Pós-Graduação Stricto Sensu, acredita-se que todas as decisões de seus gestores estão diretamente relacionadas ao alcance de um objetivo: a classificação de seus cursos em nível de excelência pela CAPES. As avaliações da CAPES são realizadas periodicamente, cabe aos gestores de Stricto Sensu, utilizar-se de instrumentos para atingir o nível de excelência. Faz-se então necessária a formulação e a implantação de estratégias para gerir com eficiência, os Programas com o intuito de alcançar o objetivo, que é a classificação de seus cursos em nível de excelência. Nesse contexto, é possível utilizar-se de um sistema de inteligência voltado para atender às expectativas acadêmicas, usufruindo assim da contribuição dos conceitos, definições e direcionamentos oferecidos e contextualizados pelo SIC. Então, para vencer as barreiras e assegurar a excelência na gestão dos PPSS, o SIC atenderá aos requisitos acadêmicos sendo denominado como Sistema de Inteligência Acadêmica (SIAc). Acredita-se que um SIAc, em PPGSSAs, poderá contribuir para a formulação e implementação das estratégias delineadas pelos Programas e para interpretação dos critérios das diversas áreas do conhecimento, principalmente com relação ao entendimento minucioso dos critérios de avaliação.

\section{Procedimentos Metodológicos}

Considerando os objetivos do trabalho desenvolvido, a pesquisa foi empírica, de caráter exploratório, com método qualitativo e de estudo de casos múltiplos, realizado no Programa em Administração da Universidade Nove de Julho (UNINOVE), IES privada, e da Universidade de São Paulo (USP), IES pública, ambas localizadas na capital de São Paulo. 
Justifica-se a adoção da definição desse desenho de pesquisa e de estudo de casos múltiplos como estratégia de investigação, uma vez que os Programas de Pós-Graduação em Administração da UNINOVE e da USP possuem indícios de utilização, ainda que informal, de Inteligência Acadêmica, como mecanismo de gestão dos Programas. Segundo Yin (2005, p. 4), o estudo de caso é um método de pesquisa exploratória e qualitativa que consiste em

[...] uma pergunta empírica que investiga um fenômeno contemporâneo no seu contexto real, quando os limites entre o fenômeno e o contexto não estão claramente evidentes e nas quais múltiplas fontes de evidências são usadas.

Neste estudo, a amostra foi intencional. Assim, as duas IES foram selecionadas tendo em vista critérios de qualidade, ou seja, o fato de serem reconhecidamente conceituadas em seu meio, e se constituírem os dois PPGSSAs com cursos conceituados na área de administração na cidade de São Paulo, SP. Por sua vez, os sujeitos sociais da pesquisa foram selecionados levando-se em conta o conhecimento e a experiência na função desempenhada por eles no desenvolvimento das estratégias e na evolução da qualidade dos Programas, considerando os parâmetros e indicadores do sistema de avaliação da CAPES. Observando esta intencionalidade, foram entrevistados em cada Programa de Pós Graduação Stricto Sensu em Administração um gestor, dois líderes de linha de pesquisa e dois especialistas técnicos, em um total de dez pessoas. A seleção desses indivíduos partiu da premissa de que eles forneceriam informações importantes, já que suas funções e atividades influenciam diretamente nas estratégias e no processo de tomada de decisão dos Programas.

Para o melhor entendimento da organização dos PPGSSAs foram utilizados na pesquisa os dados secundários obtidos por levantamento documental, mediante consulta aos documentos do Programa, como: cartas, memorandos, comunicados, agendas, planos, propostas, relatórios, registros dos sistemas Coleta/CAPES e portais do Programa e da CAPES. Para coleta de dados junto aos sujeitos sociais da pesquisa foi adotada a técnica de entrevista semiestruturada. A pauta para a realização das entrevistas guardou semelhança como a revisão de literatura empreendida, sendo que enfatizou questões abertas relacionadas com o tem a em estudo, mais precisamente: Inteligência Acadêmica e Sistema de Inteligência Acadêmica. As informações provenientes das entrevistas foram gravadas, com autorização dos respondentes, havendo ainda 
suporte de anotações das conversas mantidas com os mesmos, informalmente, e que complementam a coleta de dados, por ocasião das visitas do pesquisador àquelas instituições, Também nessas ocasiões foi realizada a observação não participante, para complementação da coleta dos dados. As entrevistas gravadas foram transcritas para análise e interpretação dos dados, tomando-se como base diretiva o que preconiza a técnica de análise de conteúdo proposta por Bardin (2002).

\section{Resultados da Pesquisa}

Os Programas, tanto da UNINOVE quanto da USP, possuem praticamente a mesma estrutura, em se tratando de coleta de informações. Ambos os gestores informaram não possuir um sistema formal de Inteligência Acadêmica. Os Programas possuem um sistema de coleta de informações, caracterizado como "Coleta/CAPES" que é um sistema de agrupamento de informações que se destina, exclusivamente, à coleta de informações necessárias para o preenchimento dos formulários de avaliação CAPES. Ambos os Programas informaram contar com uma equipe técnica reduzida para o trabalho de coleta das informações que ampara o sistema Coleta/CAPES, suficiente apenas para prospectar e suprir esse sistema com as informações necessárias. É de consenso comum entre os dois gestores que a principal fonte de informações para os dados de desempenho dos Programas é o Currículo Lattes, plataforma disponibilizada na internet pelo Conselho Nacional de Desenvolvimento Científico e Tecnológico (CNPq). A forma de uso dos Programas das informações contidas na plataforma Lattes do CNPq é para suprir o Coleta/CAPES com as informações exigidas, mas não são de fato utilizadas para sustentar um Sistema de Inteligência Acadêmica dos Programas.

Outro aspecto do processo de coleta e gestão das informações refere-se à função das pessoas especializadas para realizar os procedimentos de busca, análise e interpretação dos dados. Os respondentes indicaram haver pessoas especializadas na manipulação dos dados para cumprir especificamente as exigências institucionais da CAPES. Em nenhum dos dois Programas foi confirmada a utilização de especialistas para busca, análise e interpretação de informações que suprissem as necessidades e objetivos estratégicos dos Programas.

Todos os entrevistados indicaram em suas falas não haver no Programa uma definição clara acerca da Inteligência Acadêmica, mas entenderam e in- 
dicaram o uso dos processos de IA. As respostas, porém, não configuram um conceito claro sobre Inteligência Acadêmica, mas dão uma noção da prática de seus processos. Assim, intuitivamente, ou pela prática administrativa dos Programas, as coordenações e os envolvidos de alguma forma com a gestão desses Programas utilizam-se de procedimentos, ainda que de forma simples e não sistematizada, de IA. Podem-se aceitar algumas razões para esse estágio de entendimento da Inteligência Acadêmica junto ao setor administrativo dos PPGSSAs, visto a IA ser ainda inusitada no meio gerencial acadêmico.

Outras formas de uso da Inteligência Acadêmica também foram identificadas, como o monitoramento dos agentes reguladores da Pós-Graduação Stricto Sensu (CAPES) e Ministério da Educação (MEC). E, da mesma forma, apesar de não estarem sendo usada estruturalmente nos Programas, a prática de seus processos é comum e rotineira. Por exemplo, os gestores dos Programas indicaram não fazer monitoramento de ações regulatórias, mas realizam comparativos de cumprimento de requisitos e das condições reguladoras da finalidade dos Programas por ocasião do envio dos relatórios anuais das atividades dos Programas.

Os gestores dos PPGSSAs indicaram também não realizarem monitoramento das ações de órgãos regulatórios, demonstrando não haver uma preocupação preventiva ou antecipatória formalizada para eventos com influência sobre o desempenho de seus Programas, como os requisitos e padrões de qualidade, exigidos dos PPGSSAs e impostos por tais órgãos, que são utilizados para orientar o desempenho individual dos docentes-pesquisadores de seus respectivos Programas. A forma de utilização dos padrões, porém, parece induzir a uma dinâmica meramente corretiva, não preventiva ou antecipatória, na gestão dos Programas. No Programa da USP, mais especificamente, tais padrões foram citados pelo gestor, como fundamento de objetivos de melhoria da qualidade do Programa, que inclui o planejamento das atividades realizadas.

Também se buscou identificar o papel/finalidade da Inteligência Acadêmica nos Programas. Nenhum dos entrevistados, em ambos os Programas, apontou um papel específico para a Inteligência Acadêmica, já que os Programas não reconhecem formalmente sua existência, mas indícios de sua aplicação. No entendimento dos entrevistados, no entanto, a IA serviria para coletar as informações dos órgãos reguladores, interpretados em termos de suas implicações (feitos pelos coordenadores nos colegiados) para o dia-a-dia das atividades dos Programas e disseminá-las junto aos seus docentes. Em relação ao processo do Sistema de Inteligência Acadêmica, conforme será mostrado na Figura 3, 
há compreensão dos entrevistados sobre o papel/finalidade da Inteligência Acadêmica nos PPGSSAs, sendo este o de coletar, gerar um produto, por meio da interpretação das informações e disseminá-las nos PPGSSAs.

Também foi houve estudo sobre o formato de gestão dos PPGSSAs e, ao mesmo tempo, a visualização de indícios da Inteligência Acadêmica e suas possíveis contribuições nesses Programas. Os gestores verbalizaram e descreveram as características de gestão dos seus Programas, que se mostraram, de fato, diferenciadas em estilo e modo de operação. No caso da USP, o Programa discute e propõe em pequeno comitê, os objetivos estratégicos que são posteriormente compartilhados com os docentes do Programa, nas reuniões do colegiado. É nítido o esforço do grupo na análise e interpretação dos parâmetros de qualidade da CAPES e suas implicações para as metas de desempenho do Programa. Pode-se inferir nesse esforço a interpretação e eventuais discussões de informações de benchmark de outros Programas. A presença, ainda que restrita da Inteligência Acadêmica na gestão do Programa decorre, talvez, do estilo de gestão mais estratégica desse Programa. No Programa da UNINOVE, o estilo de gestão parece ser mais aberto e menos claro, sob o ponto de vista de definição de metas e objetivos estratégicos. As atividades e a produção acadêmica são geridas conforme as oportunidades que aparecem, criando um grau de ampla flexibilidade nas metas e no direcionamento do Programa. Nesse caso é menos perceptível a presença e o uso da Inteligência Acadêmica como forma de direcionar atividades, identificar oportunidades antecipadamente e construir capacidades internas, apesar de notar-se igual esforço para interpretação das implicações dos parâmetros e padrões de qualidade exigidos pela CAPES.

Quantos aos especialistas técnicos e líderes de linha de pesquisa entrevistados, todos atribuem a forma e o estilo gerencial dos Programas, a cada gestor. Compreendem esses respondentes que é possível utilizar-se um Sistema de Inteligência Acadêmica como mecanismo para equilibrar e vencer as barreiras de competência e garantia de excelência de desempenho dos PPGSSAs. Concordaram, claramente, que um SIAc (Figura 3) poderá contribuir para a formulação e implementação das estratégias delineadas pelos Programas e para a interpretação dos critérios das diversas áreas do conhecimento, principalmente com relação ao melhor entendimento dos critérios de avaliação. Há, inclusive, verbalizações destes lideres de linha de pesquisa que evidenciam a compreensão de que o alicerce da eficácia e da sustentabilidade das estratégias 
formuladas para os Programas está diretamente relacionado com a qualidade da informação que alimenta seus processos decisórios. Há ainda entendimento por parte destes mesmos respondentes de que o processo de formulação de objetivos e de construção de estratégias dos Programas é fundamentado no plano estratégico, cujas origens se reportam à missão, visão e vocação dos respectivos Programas, numa adaptação da lógica de Almeida (2003), para a elaboração de planos estratégicos. $\mathrm{E}$, de que as ações estratégicas resultantes devem influenciar os resultados do desempenho dos Programas de forma satisfatória ou insatisfatória. Os fatores de insatisfatoriedade, igualmente, decorrem dos padrões incorporados nos próprios critérios de qualidade definidos pela CAPES. É compreensível, com tais resultados, afirmar que os Programas concentrem-se, exclusivamente, no alcance dos padrões mínimos de desempenho/qualidade incorporados naqueles critérios. Atingir tais padrões, com base exclusiva nos recursos e nas capacidades internas, sem abordagens alternativas, parece ser menos eficiente do que considerar modelos, soluções e caminhos alternativos como os oferecidos por um SIAc. Na Figura 3, que propõe os mecanismos de apoio do SIAc aos PPGSSAs, houve o entendimento por parte dos gestores de que esse sistema teria a função formal de prospectar informações, modelos, soluções, caminhos e estratégias alternativas passíveis de serem utilizadas pelos Programas. Dessa forma, com a contribuição do SIAc, o processo decisório para as ações administrativas no desenvolvimento das capacidades internas, na alocação otimizada de recursos, a simplificação de processos e o necessário ajustamento do modelo dos Programas seriam altamente beneficiados.

Da mesma forma os objetivos, metas e correspondentes ações seriam melhor postulados e as estratégias construídas de forma deliberada ou emergente poderiam tornar-se mais eficazes para o desempenho produtivo dos Programas e a qualidade geral dos seus serviços. 


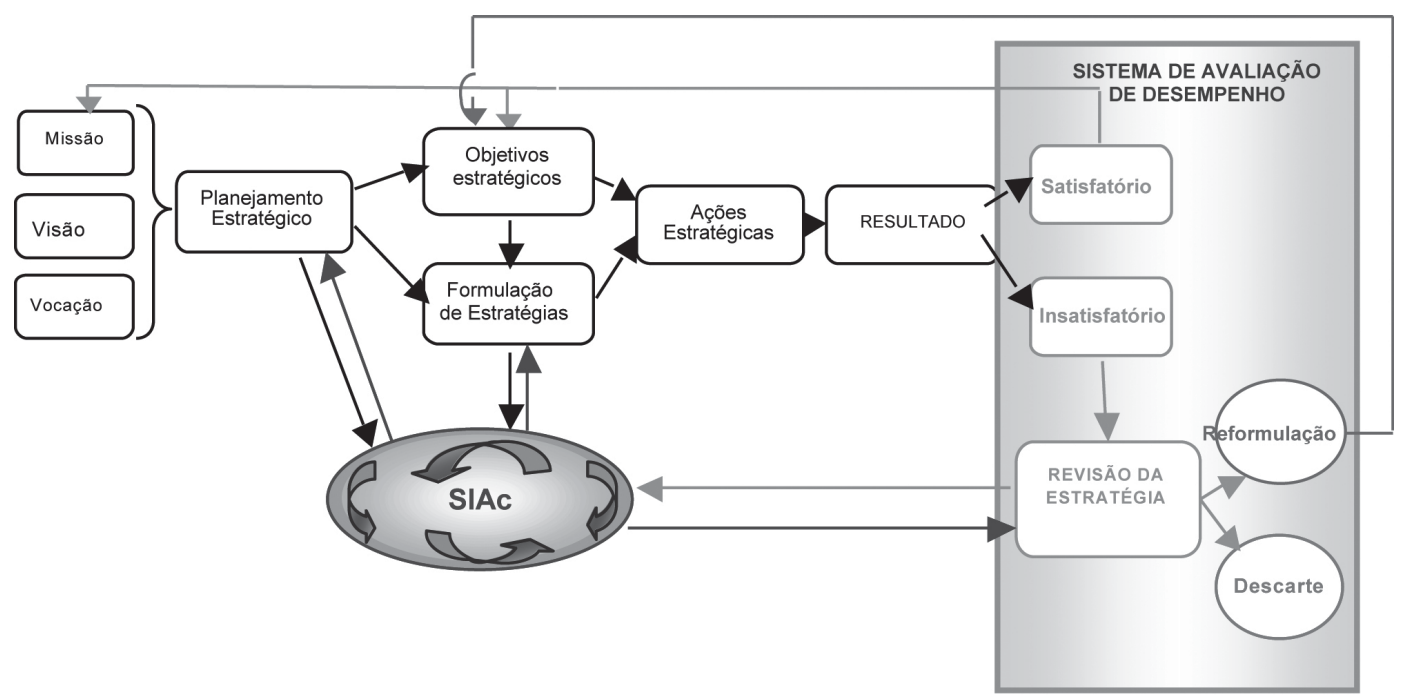

Figura 3: A função do SIAc como mecanismo de apoio aos PPGSSAs

Fonte: Elaborada pelos autores deste artigo com base nos dados da pesquisa

A análise do ambiente da gestão dos dois Programas mostra a existência de um espaço crítico para uma importante função administrativa, a Inteligência Acadêmica. A Figura 4 mostra como um SIAc poderia dar suporte às atividades de gestão pertinentes aos PPGSSAs, e não somente àquelas atividades relativas ao preenchimento do Coleta CAPES. O desenvolvimento de um SIAc teria a função formal de prospectar informações, modelos, soluções, caminhos e estratégias alternativas passíveis de serem utilizadas para uma gestão estratégica dos Programas. Assim, é importante que o sistema contenha mecanismos para garantir essencialmente três fundamentos: (a) identificação das necessidades de informação; (b) forma de prospecção; e (c) geração de um produto da prospecção que possa ser útil às estruturas administrativas e técnicas dos Programas. Com base nesses princípios, propõe-se o modelo processual de um SIAc, como aparece na Figura 4.

Nesse modelo processual assume-se como premissa básica que os PPGSSAs possuem um Plano Estratégico que direciona seus esforços de aperfeiçoamento da qualidade e crescimento do Programa. Nesse caso, o Plano Estratégico seria o principal fornecedor de demandas de informações para o Programa. 


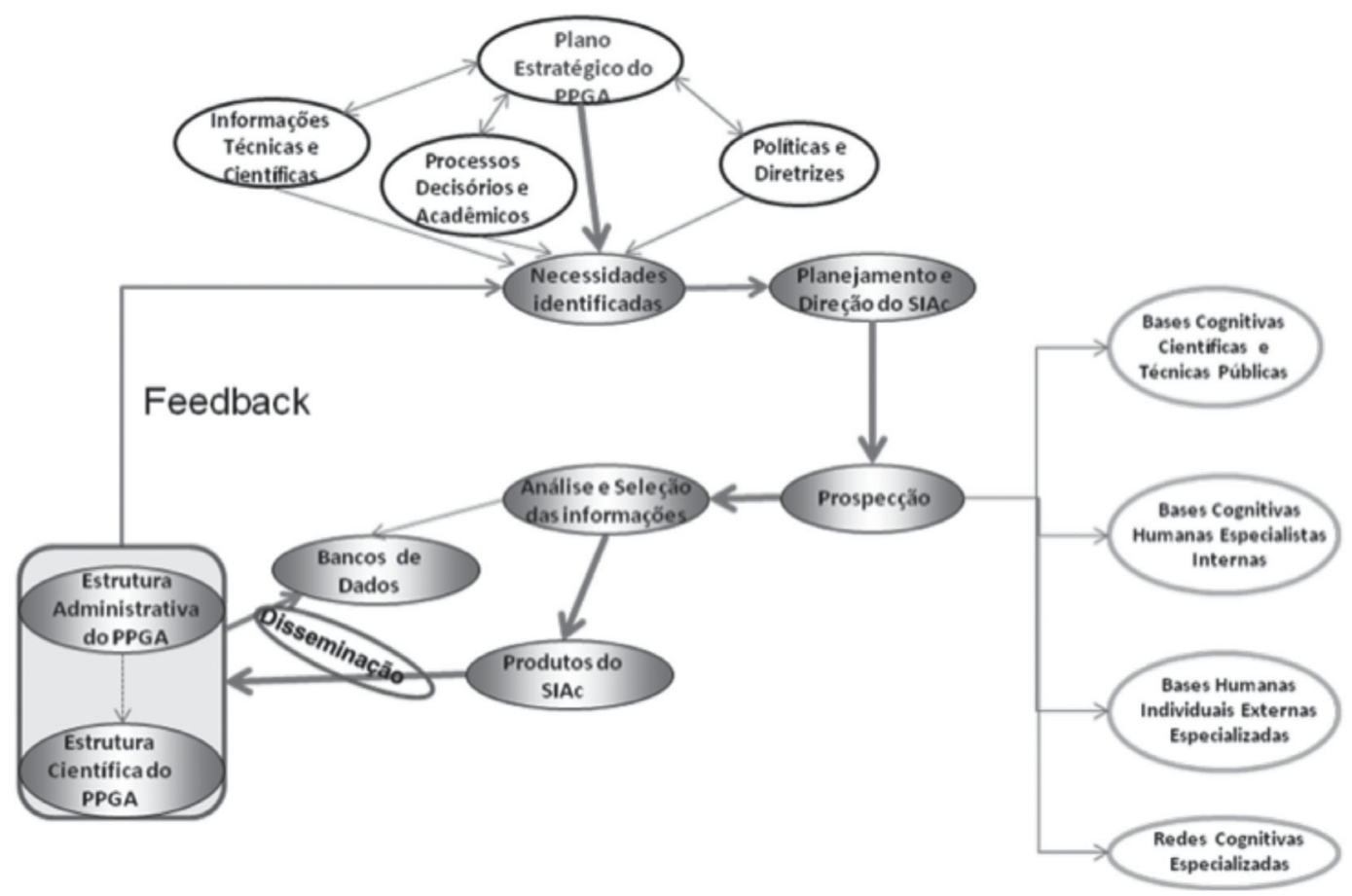

Figura 4: Modelo Processual do SIAc

Fonte: Elaborada pelos autores deste artigo com base nos dados da pesquisa

As necessidades de informações são identificadas a partir de diversas solicitações que podem ter origem nas informações técnicas e científicas, nos processos decisórios acadêmicos e nas políticas e diretrizes institucionais dos Programas, integralizadas por meio dos objetivos estratégicos expressos naqueles Planos Estratégicos. As informações técnicas e científicas compreendem as de caráter administrativo, como informações solicitadas pela CAPES e por outras Agências de fomento, bem como informações técnicas de interesse dos pesquisadores do Programa.

\section{Conclusão}

Há entendimento por parte dos sujeitos sociais da pesquisa, tanto do gestor, como dos dois líderes de linha de pesquisa e dos dois especialistas técnicos, em cada Programa pesquisado, em um total de dez pessoas entrevistadas, que os processos decisórios e os acadêmicos compreendem as solicitações ou 
necessidades de informações da rotina administrativa dos PPGSSAs. Também há entendimento de que as políticas e diretrizes demandam informações que justificam a dinâmica, o direcionamento, o nível de qualidade e os objetivos de visibilidade institucional dos Programas. Da mesma forma, há compreensão de que o SIAc pode funcionar adequadamente neste contexto, dependendo, entretanto, do direcionamento que o plano estratégico propuser ao Programa. Para tanto, há consenso da necessidade de determinação anterior do lugar onde o Programa quer chegar, para saber que tipo de informação precisa para tomar decisões acertadas, alocar racionalmente seus recursos e desenvolver eficientemente suas competências e capacidades. Os entrevistados argumentaram, inclusive, de que se o Programa não tiver claro aonde quer chegar, o SIAc será pouco efetivo e o Programa perderá em qualidade e, muito provavelmente, em desempenho geral. Há entendimento claro da relação entre o Planejamento Estratégico, a Estratégia, a Inteligência Acadêmica e o SIAc. Há compreensão suficiente, com base nos resultados da pesquisa, de que a prospecção e coleta dos dados requeridos para alimentação do SIAc devem ser feitas nas bases cognitivas científicas e técnicas públicas (sites ou documentos de Agências oficiais, como a CAPES, Agências de fomento, Fundações de Apoio, entre outros); nas bases cognitivas humanas especializadas internas (pessoal técnico especializado dentro dos PPGSSAs); nas bases cognitivas humanas externas especializadas (coordenadores de outros PPGSSAs, técnicos, administradores e gerentes das Agências, Fundações, etc.) e redes cognitivas especializadas (grupos de pesquisa, pesquisadores que participam de eventos ou encontros das várias áreas de conhecimento). Da mesma maneira pode-se concluir de que as informações obtidas pelo processo de prospecção serão analisadas e selecionadas segundo os requisitos das demandas, gerando assim uma ou mais bases de dados e um produto de informações específicas de acordo com a demanda original, gerado pela equipe do SIAc. Essas informações (bases de dados e os produtos específicos) devem ser disseminadas para as estruturas científicas e administrativas do PPGA.

Pode-se concluir ainda que não apenas os produtos do SIAc devem alimentar as estruturas decisórias de um PPGSSA. Os bancos de dados, como principal produto do SIAc, construídos ao longo das diversas prospecções, também podem ser disponibilizados para acesso direto por pessoas ou grupos dentro do Programa, em momentos e de acordo com suas necessidades.

A contribuição do SIAc é compreendida pelos sujeitos sociais do estudo como necessária e crítica, visando amparar e nortear os processos decisórios, 
as ações administrativas no desenvolvimento das competências e capacidades internas, a locação otimizada de recursos, a simplificação dos processos internos e a construção de uma dinâmica interna mais eficiente nos Programas.Com a adoção e implantação do SIAc há ideia de que o PPGSSAs poderiam desenvolver propostas de crescimento e excelência, de forma autônoma com maior qualidade e mais sintonizadas com suas características e com as do meio em que opera. Além disso, tais propostas, ao mesmo tempo em que respondem aos requisitos de qualidade da CAPES, poderiam determinar uma nova dinâmica, em ritmo compatível, de crescimento para os Programas.

A conclusão final é de que os Programas podem expandir suas capacidades técnicas e científicas, aperfeiçoar o uso de seus recursos e ampliar suas competências centrais, por meio da adoção do SIAc. Isso seria feito de forma direcionada e equilibrada com seus interesses, afinados com o entorno em que operam e com a preocupação de retorno à sociedade. Finalmente, pode-se afirmar que há entendimento nesta pesquisa de que os Programas que formalizarem o SIAc têm expectativas mais promissoras de determinar o tipo e as áreas de visibilidade externos que almejam, assim como a função e o papel do próprio Programa na Instituição ao qual esteja vinculado.

\section{Academic Intelligence System for Management of Graduate Business Programs}

\section{Abstract}

The aim of this work was to analyze the management of two Business Graduate Programs (PPGSSAs), seeking to contribute to the implementation of a SIAC, as a support to the decision making process and to the formulation of strategies in the surveyed programs. The PPGSSAs selected are incorporated by their respective Higher Education Institutions (HEI), one public and one private. They were intentionally selected based on quality criteria. The theoretic fundament involved, focused especially the literature on Competitive Intelligence $(\mathrm{CI})$ and the interface with strategies formulation. The empirical research was exploratory, using qualitative methods, and multiple case studies. The results indicate that in both programs there is evidence of the academic 
intelligence (AI) presence, albeit in a simplified and targeted way, oriented to meet the quality requirements imposed by CAPES. The analysis allowed for pointing out the role and functions of a SIAC, within the administrative and operational structure of the Programs studied, where it supports and enhances the formulation of strategies to improve quality, increase visibility and social integration of both Programs studied. There is also understanding by the academic managers, by the research line leaders and by the technical expert participants in the research on the opportunity to adopt/ implement a SIAC in their PPGSSAs.

Key words: Competitive Intelligence. Strategic Management. Business Graduate Programs. Academic Intelligence. Academic Intelligence System (IACS).

\section{Referências}

ABRAIC. Associação Brasileira dos Analistas de Inteligência Competitiva. [2010?]. Disponível em: <http://www.abraic.org.br/site/faqs.asp > . Acesso em: 25 jul. 2010.

ALMEIDA, Martinho Isnard Ribeiro de. Manual de planejamento estratégico: desenvolvimento de um plano estratégico com a utilização de planilhas Excel. 2. ed. São Paulo: Atlas, 2003.

BALESTRIN, Alsones. Inteligência competitiva nas organizações. In: WORKSHOP DE INTELIGÊNCIA COMPETITIVA E GESTÃO DO CONHECIMENTO, 2, Florianópolis, Paulo, 2001. Anais... Florianópolis, UFSC, 2001.

BARDIN, Laurence. Análise de conteúdo. Lisboa: Edições 70, 2002.

BATTAGLIA, Maria da Glória Botelho. A inteligência competitiva modelando o sistema de informação de clientes - Finep. Ciência da Informação, Brasília, v. 28, n. 2, 1999.

BESSA, Jorge. Inteligência competitiva: uma necessidade no mundo globalizado. [2010?]. Disponível em: <http://www.abraic.com.br>. Acesso em:3 maio 2010. 
BIAGI, Helio; BERJEAUT, Guy Pierre F. A inteligência competitiva como suporte ao planejamento estratégico nas instituições financeiras brasileiras. Revista Hispana de La Inteligencia Competitiva - Puzzle, España, v. 6, n. 23, p. 47- 60, agosto/ oct. 2006.

COELHO, Gilda M. et al. Ensino e pesquisa no campo da inteligência competitiva no Brasil e a cooperação franco-brasileira. Revista Hispana de La Inteligencia Competitiva - Puzzle, España, v. 6, n. 23, p. 12-19,agosto/oct. 2006.

COMAI, Alessandro. Global code of ethics and competitive intelligence purposes: an ethical perspective on competitors. Journal of Competitive Intelligence and Management, Windsor, v. 2, n. 1, p. 25-44, 2004.

COMBS, Richard E. The competitive intelligence handbook. Metuchen: Scarecrow, 1992.

CONNOLLY, Kate Phillips et al. International supply chain management: a walk around the elephant. Irish Journal of Management. Dublin, v. 26, n. 1, p. 149168, 2005.

CORREIA, C. C. Getting competitive. Library Journal. New York, v. 131, n. 7, p. 52-54, 2006.

DRUCKER, Peter F. Introdução à administração. São Paulo: Pioneira, 1984.

FISHMANN, Adalberto Américo; ALMEIDA, Martinho Isnard Ribeiro de.

Planejamento estratégico na prática. 2. ed. São Paulo: Atlas, 1991.

FLEISHER, Craig S.; WRIGHT, Sheila; TINDALE, Robb. Bibliography and assessment of key competitive intelligence scholarship: part 4 (2003-2006). Journal of Competitive Intelligence and Management, Windsor, v. 4, n. 1, p. 34-107, 2007.

FULD, Leonard M. The new competitor intelligence: the complete resource for finding, analyzing, and using information about your competitors. New York: John Wiley \& Sons, 1995.

GOMES, Elisabet Braz P.; BRAGA, Fabiane dos Reis. Inteligência Competitiva no Brasil: uma realidade corporativa. Revista Hispana de La Inteligencia Competitiva - Puzzle, España, v. 6, n. 23, p. 5-10, agosto/oct. 2006. 
HERRING, Jan P. Key intelligence topics: a process to identify and define intelligence needs. Competitive Intelligence Review, New York: v. 10, n. 2, p. 4-14,1999.

KAHANER, Larry. Competitive intelligence: how business gathers, analyze, and use information to succeed in the global marketplace. New York: Simon \& Schuster, 1996.

KOTLER, Philip; ARMSTRONG, Gray. Princípios de marketing. Rio de Janeiro: Prentice- Hall, 1993.

MILANO, Camilo Ricardo. Relaciones entre la inteligencia estratégica y el análisis estratégico. Inteligencia total, Chile, v. 1 n. 1, p. 24-35, mar. 2004.

MILLER, Jerry P. O milênio da inteligência competitiva. Porto Alegre: Bookman, 2002.

MINTZBERG, Henry. Os 5 "Ps" da estratégia. In: MINTZBERG, Henry; QUINN, James Brian. O processo da estratégia. 3. ed. Porto Alegre: Bookman, 2001.

MINTZBERG, Henry, QUINN, J. B. O processo da estratégia. Porto Alegre: Bookman, 2001.

PORTER, Michael E. Competição: oncompetition. Rio de Janeiro: Campus, 1999. 1996. . What is strategy? Harvard Business Review. Boston, p. 61-78, Nov./Dec.,

PRESCOTT, John E.; MILLER, Stephen. H. Inteligência competitiva na prática. Rio de Janeiro: Campus, 2002.

RODRIGUES, Leonel Cezar; RICCARDI, Riccardo. Inteligência competitiva: nos negócios e organizações. Maringá: Unicorpore, 2007.

RODRIGUES, Leonel Cezar. Technical competitive intelligence system: an innovation and technology management tool. In: DELENER, Nejdet (Ed.) Service science research, strategy, and innovation: dynamic knowledge management methods. New York: IGI Global, 2011. p. 172-210.

SAMMON, W. L.; KURLAND, M. A.; SPITALNIC, R. Business competitor intelligence: methods for collecting, organizing and using information. New York: John Wiley \& Sons, 1984. 
SCIP - Society of Competitive Intelligence Professionals. [2010?]. Disponível em: $<$ http://www.scip.org/index.php>. Acesso em: 10 jul. 2010.

SILVA, Alexandre R. Concepção de um sistema de inteligência competitiva para uma organização do setor de insumos e bens de produção do complexo agroindustrial. Dissertação (Mestrado em Engenharia da Produção) Programa de Pós-Graduação em Engenharia de Produção, Universidade Federal de São Carlos, São Carlos, 2005.

TYSON, Kirk W. M. The complete guide to competitive intelligence. 2. ed., Chicago: Leading Edge Publ., 2002.

WEISS, Arthur. A brief guide to competitive intelligence: how to gather and use information on competitors. Business Information Review, London, v. 19, n. 2, p. 38-47, Jun. 2002.

YIN, Robert K. Estudo de caso: planejamento e métodos. 3. ed. Porto Alegre: Bookman, 2005.

ZACCARELLI, Sérgio Baptista. Estratégia e sucesso nas empresas. São Paulo: Saraiva, 2002. 